\title{
Methotrexate: the gold standard without standardisation
}

\author{
Jonathan Kay, ${ }^{1}$ Rene Westhovens ${ }^{2}$
}

Even in the current era of biological targeted therapies, methotrexate (MTX) remains the initial preferred antirheumatic drug and is widely prescribed for patients with rheumatoid arthritis (RA). The combination of its perceived efficacy, acceptable safety profile and low cost, as well as decades of clinical experience with its use that have been accumulated by current and previous generations of rheumatologists, make MTX the cornerstone of treatment for RA and the anchor drug for combination with each of the newer biological agents.

Four papers in this journal ${ }^{1-4}$ provide general guidance for the use of MTX, its optimal dose and route of administration and its potential toxicity (see articles on pages 1086, 1094, 1100 and 1105). This "3E Initiative," supported by an unrestricted educational grant from Abbott, began with an extensive systematic literature review (Evidence) that was discussed by each of 17 national scientific committees (Expertise). These national groups combined to form a broad international panel of experts who shared (Exchange) the individual national recommendations. While acknowledging changes in MTX use over time and in different geographical regions, the authors propose consensus guidelines for the use of MTX to manage patients with RA.

\section{EVIDENCE}

Of the "Evidence" accumulated by the authors' search of the medical literature, nearly half $(9 / 19)$ of the studies of MTX use that were included in their metaanalysis had been published during the last decade of the 20th century. ${ }^{4}$ The authors acknowledge that, during this era, MTX was prescribed at lower doses than are currently used. Also, folic acid

\footnotetext{
${ }^{1}$ Division of Rheumatology, Allergy, and Immunology, Department of Medicine, Massachusetts General Hospital and Harvard Medical School, Boston, Massachusetts, USA; ${ }^{2}$ Division of Rheumatology, Department of Musculoskeletal Sciences, UZ Gasthuisberg, KU Leuven, Belgium
}

Correspondence to: Professor R Westhovens, Division of Rheumatology, Department of Musculoskeletal

Sciences, UZ Gasthuisberg, KU Leuven, B-3000 Leuven, Belgium; rene.westhovens@uz.kuleuven.ac.be supplementation was not administered as systematically and MTX doses were escalated less rapidly than is the current custom. In addition, the disease-modifying antirheumatic drugs (DMARDs) with which MTX was combined in these studies included DMARDs not commonly used in current clinical practice (such as intramuscular gold, penicillamine, bucillamine, ciclosporin A, doxycycline and levofloxacin) and did not include biological targeted therapies. Thus, the evidence upon which these guidelines are based does not completely reflect contemporary practice.

Because studies comparing MTX monotherapy with tumour necrosis factor (TNF) antagonist monotherapy and with the combination of MTX and a TNF antagonist in early or recent-onset $\mathrm{RA}^{5}{ }^{6}$ were not included in the meta-analysis, recommendation 7 about "combination therapy" is thereby limited to combinations of MTX with non-biological DMARDs - a treatment approach that now, with biological targeted therapies available, is not often used. The authors also do not examine the use of MTX in "step-down" treatment strategies in patients with early RA.?

The 17 countries represented by the multinational group are predominantly European, with representatives from Australia, North America (Canada, but not the United States), Central America (Mexico) and South America (Brazil). Notably absent are representatives from Africa and Asia. The data presented in the literature from clinical trials are derived mostly from European and North American patients. However, in attempting to reach broad consensus, the authors fail to look for evidence of differences in MTX use among specific patient populations. For example, lower MTX doses are used by Japanese patients with RA (where the maximum MTX dose is restricted by law to $8 \mathrm{mg} /$ week $)^{8}$ than by patients of European and African ancestry. Pharmacogenetic differences among different ethnic groups ${ }^{9}$ should be evaluated and included in "multinational evidencebased recommendations for the use of MTX in rheumatic disorders."

\section{EXPERTISE}

Although the authors claim to make "evidence-based recommendations," evidence appears to have been over-ruled by eminence (or so-called "expert opinion") in some circumstances, without clear justification. One example is found in the recommended evaluation for patients starting treatment with MTX (recommendation 1), in which "contraindications to MTX use frequently listed" as exclusion criteria "in randomised clinical trials" of treatment for patients with RA are added to the list of risk factors that are justified by data published in the peerreviewed literature. Although it is prudent to obtain lipid studies in all patients with $\mathrm{RA}$, because of the increased risk of cardiovascular disease in this patient population, ${ }^{10}$ it is not clear why this is relevant to "the work-up for patients starting MTX" (recommendation 1). Only by performing the necessary studies to fill the gaps in evidence can the role of "Eminence" (the fourth E) be minimised in the development of future recommendations.

Where evidence from the peer-reviewed literature is insufficient to support recommendations for clinical practice, it must be supplemented by "expert opinion." An example is the "eminence-based" proscription of MTX use in male patients with RA "for at least 3 months before planned pregnancy" and in female patients with RA during breast feeding in recommendation 10. Although these recommendations are prudent and consider patient safety, such gaps in evidence should prompt the collection of relevant observational data from the several existing large registries of patients with RA.

The members of the "broad international panel of rheumatologists" from 17 countries on four continents possess broad and diverse experience in managing patients with RA and thus provide diverse expertise in this practice. It is unfortunate that the publications from the " $3 \mathrm{E}$ Initiative" report only the consensus and do not also present the wide variety of experience among rheumatologists in different countries and in different practice settings (academic versus community based).

\section{EXCHANGE}

The third E (Exchange) in this "3E Initiative," although not described extensively in these papers, reflects the discussion of ideas and recommendations among rheumatologists. Thus doctorcentred, risk factors and safety issues are 
identified. Others, such as nausea, hair loss and fear of unknown drug toxicities, are of paramount importance to many patients, yet are not dealt with. The better tolerability of MTX, when used in combination with TNF antagonists in a step-down rather than in a step-up protocol in early RA, is important to the patient and needs to be dealt with in recommendations of MTX use. ${ }^{11}$

Because the patient is the focus of the therapeutic doctor-patient relationship, we urge that patients be included in the process of "Exchange." The source of the assertion, in these papers, that oral MTX is widely preferred by patients is not documented; other possible routes of MTX self-administration, such as subcutaneous injection, should also be discussed. Recommendation 1 mentions the need for patient education, but does not suggest how this should be accomplished.

Implementation of these recommendations and guidelines can certainly benefit from the perspectives and ideas of patients. As has recently become a principle in the development of EULAR recommendations ${ }^{12} 13$ and OMERACT initiatives, ${ }^{14}$ we advocate that patients should be involved, preferably from the beginning, in future initiatives to develop consensus treatment recommendations.

\section{CONCLUSIONS AND SUGGESTIONS FOR A RESEARCH AGENDA}

In summarising the available evidence that supports the use of MTX in treating RA, the "3E Initiative" papers in this journal highlight the disproportionate number of randomised, controlled clinical studies of the newer, more expensive targeted biological therapies compared with the number of those designed to study MTX. The most extensive data on the use and safety of MTX have been derived from the large-scale randomised, controlled clinical studies of biological agents used in combination with MTX, in which the "placebo" arm has been MTX monotherapy.

The observation that, among patients with early RA the combination of MTX with a TNF antagonist appears to be associated with fewer side effects than MTX monotherapy illustrates the complexity of factors surrounding the efficacy and tolerability of MTX. Although this effect could result from a synergy between the TNF antagonist and MTX, it also could be a consequence of the greater efficacy of combination therapy: the better a patient feels, the fewer treatment side effects he or she may notice or report.

To gather the information needed to guide the present use of MTX in patients with RA, randomised controlled clinical studies of treatment strategies that employ doses of MTX up to $25 \mathrm{mg} /$ week are needed. Such efficacy trials should also examine optimisation of MTX dose escalation strategies and schedules, standardisation of drug safety monitoring procedures and implementation of patient education protocols that take into account the perspectives of patients. Although some of this information might be derived from data collected prospectively in patient registries, confounding by indication always biases such registry data. Few of the existing registries of patients with RA currently track patients who are receiving MTX monotherapy.

Because MTX is widely used to treat RA, it ought to be possible to enrol enough patients in trials that examine the optimal design and effectiveness of different therapeutic strategies that include MTX. The international community of rheumatologists should work together to execute large, simple randomised trials, collecting data from clinical practice and sharing these data using internet-based tools, to deal with those questions regarding optimal MTX use that remain unanswered in the medical literature. Such large collaborative efficacy trials would display regional variations, which might result from pharmacogenomic differences among patients or from attitudinal differences among rheumatologists. Although it is unlikely that the pharmaceutical industry would support clinical studies of a generic drug, such as MTX, support for these initiatives might be provided by government-run scientific research institutes, government health insurers, third-party payers, patient organisations, or professional organisations of rheumatologists. By filling in the gaps in our knowledge about the use of MTX in treating RA, we will be able to optimise the use of this "gold standard" in clinical practice and improve the wellbeing of our patients with RA.

\section{Competing interests: None.}

Accepted 23 February 2009

Ann Rheum Dis 2009;68:1081-1082.

doi:10.1136/ard.2008.102822

\section{REFERENCES}

1. Visser K, Katchamart W, Loza E, Martinez-Lopez JA Salliot C, Trudeau J, et al. Multinational evidencebased recommendations for the use of methotrexate in rheumatic disorders with a focus on rheumatoid arthritis: integrating systematic literature research and expert opinion of a broad international panel of rheumatologists in the $3 E$ Initiative. Ann Rheum Dis 2009:68:1086-93.

2. Visser K, van der Heijde D. Optimal dosage and route of administration of methotrexate in rheumatoid arthritis: a systematic review of the literature. Ann Rheum Dis 2009;68:1094-9.

3. Salliot C, van der Heijde D. Long-term safety of methotrexate monotherapy in patients with rheumatoid arthritis: a systematic literature search. Ann Rheum Dis 2009;68:1100-4.

4. Katchamart W, Trudeau J, Phumethum V, Bombardier C. Efficacy and toxicity of methotrexate (MTX) monotherapy versus MTX combination therapy with non-biological disease-modifying antirheumatic drugs in rheumatoid arthritis: a systematic review and meta-analysis. Ann Rheum Dis 2009;68:1105-12.

5. Klareskog L, van der Heijde D, de Jager JP, Gough A, Kalden J, Malaise M, et al. TEMPO (Trial of Etanercept and Methotrexate with Radiographic Patient Outcomes) study investigators. Therapeutic effect of the combination of etanercept and methotrexate compared with each treatment alone in patients with rheumatoid arthritis: double-blind randomised controlled trial. Lancet 2004;363:675-81.

6. Breedveld FC, Weisman MH, Kavanaugh AF, Cohen SB, Pavelka K, van Vollenhoven R, et al. The PREMIER study: a multicenter, randomized, double-blind clinical trial of combination therapy with adalimumab plus methotrexate versus methotrexate alone or adalimumab alone in patients with early, aggressive rheumatoid arthritis who had not had previous methotrexate treatment. Arthritis Rheum 2006;54:26-37.

7. Verschueren P, Esselens G, Westhovens R. Daily practice effectiveness of a step-down treatment in comparison with a tight step-up for early rheumatoid arthritis. Rheumatology (Oxford) 2008:47:59-64.

8. Suzuki Y. Methotrexate for the treatment of rheumatoid arthritis in Japan-much more still remains to be resolved. Nippon Rinsho 2002;60:2331-8.

9. Ranganathan $\mathbf{P}$, Culverhouse R, Marsh S, Mody A, Scott-Horton TJ, Brasington R, et al. Methotrexate (MTX) pathway gene polymorphisms and their effects on MTX toxicity in Caucasian and African American patients with rheumatoid arthritis. J Rheumatol 2008;35:572-9.

10. Salmon JE, Roman MJ. Cardiovascular manifestations of rheumatologic diseases. Circulation 2007; 116:2346-55.

11. Goekoop-Ruiterman YP, de Vries-Bouwstra JK, Allaart CF, van Zeben D, Kerstens PJ, Hazes JM, et al. Clinical and radiographic outcomes of four different treatment strategies in patients with early rheumatoid arthritis (the BeSt study): a randomized, controlled trial. Arthritis Rheum 2005;52:3381-90.

12. Dougados M. EULAR efforts to define quality of care. Clin Exp Rheumatol 2007;25(Suppl 47):14-7.

13. Kiltz U, van der Heijde D, Mielants $H$, Feldtkeller E, Braun J. ASAS/EULAR recommendations for the management of ankylosing spondylitis - the patient version. Ann Rheum Dis Published Online First: 17 October 2008. doi:10.1136/ard.2008.096073.

14. Tugwell P, Boers M, Brooks P, Simon L, Strand V, Idzerda L. OMERACT: an international initiative to improve outcome measurement in rheumatology. Trials 2007; 8:38. 